\title{
KINEMATIC FORMULA AND TUBE FORMULA IN SPACE OF CONSTANT CURVATURE
}

\author{
by SUNGYUN LEE*
}

(Received 12th April 1988)

\begin{abstract}
The Euler characteristic of an even dimensional submanifold in a space of constant curvature is given in terms of Weyl's curvature invariants. A derivation of Chern's kinematic formula in non-Euclidean space is completed. As an application of above results Weyl's tube formula about an odd-dimensional submanifold in a space of constant curvature is obtained.
\end{abstract}

1980 Mathematics subject classifications (1985 Revision): 53C20, 53C65.

\section{Introduction}

Let $P$ be a compact orientable $p$-dimensional Riemannian manifold which is imbedded in $n$-dimensional non-Euclidean space $E^{n}(K)$ of constant curvature $K$ (briefly $P \subset E^{n}(K)$ ). Denote by $R_{P}$ and $R_{K}$ the curvature tensor fields of $P$ and $E^{n}(K)$ respectively.

In 1987 Ishihara [3] derived an interesting formula for the Euler characteristic $\chi(P)$. Let $p=\operatorname{dim} P$ be even. He showed that

$$
\chi(P)=\frac{1}{(2 \pi)^{p / 2}} \sum_{0 \leqq i \leqq p / 2} K^{i}(2 i-1) ! ! k_{p-2 i}\left(R_{P}-R_{K}\right),
$$

where $k_{2 c}\left(R_{P}-R_{K}\right)$ are Weyl's curvature invariants (see Section 2) and $m ! !=m(m-2) \ldots 4 \cdot 2$ or $m ! !=m(m-2) \ldots 3 \cdot 1$ according as $n$ is even or odd.

Ishihara's derivation of (1.1) relied on a Teufel's result [6]. In this article we give a simple proof of it using the exterior product of double forms and the contraction operator.

Ishihara [3] also mentioned the following result which generalizes Chern's kinematic formula in Euclidean space [1]. Let $P \subset E^{n}(K)$ and $Q \subset E^{n}(K)$ be compact submanifolds of dimensions $p$ and $q$ respectively. Let $E_{K}(n)$ be the group of proper motions of $E^{n}(K)$ and $d g$ the standard kinematic density on $E_{K}(n)$. If $0 \leqq e$ even $\leqq p+q-n$ and $g \in E_{K}(n)$, then

$$
\int \mu_{e}(P \cap g Q, K) d g=\sum_{0 \leqq i \text { even } \leqq e} c_{e, i} \mu_{i}(P, K) \mu_{e-i}(Q, K)
$$

*Partially supported by KOSEF of Korea. 
with constants $c_{e, i}$ depending on $p, q, n, e$ and $i$. Here integral invariants $\mu_{e}(P, K)$ are related to $k_{e}\left(R_{P}-R_{K}\right)$ by

$$
\mu_{e}(P, K)=\frac{2^{e / 2}(p-e) !(e / 2) !}{p !} k_{e}\left(R_{P}-R_{K}\right)
$$

The second purpose of this article is to determine constants $c_{e, i}$ which Ishihara could not do. In fact $c_{e, i}$ in (1.2) are given by

$$
c_{e, i}=O_{n+1} O_{n} \ldots O_{2} \frac{\frac{O_{p+q-n+1} O_{p+q-n+2}(e / 2) !}{O_{p+q-n-e+2}}}{\left(\frac{O_{p+1} O_{p+2}(i / 2) !}{O_{p-i+2}}\right)\left(\frac{O_{q+1} O_{q+2}((e-i) / 2) !}{O_{q-e+i+2}}\right)} .
$$

Here $O_{j}=2 \pi^{j / 2} / \Gamma(j / 2)$ is the volume of unit sphere in Euclidean $j$-space.

As an application of the formulas (1.1) and (1.2) we derive Weyl's tube formula for a compact odd-dimensional submanifold $P \subset E^{n}(K)$. This derivation shows a close relationship among the Gauss-Bonnet theorem, Chern's kinematic formula and Weyl's tube formula.

\section{New derivation of Ishihara's formula}

We refer to [2] for basic facts on double forms. We shall say that a double form $R$ of type $(2,2)$ which has the same symmetries as the curvature tensor of a Riemannian manifold $P$ is curvature-like. Let $R$ be a curvature-like tensor field on $P$. The complete contraction $C^{2 c}\left(R^{c}\right)$ of $R^{c}=R \wedge \cdots \wedge R(c$ times $)$ is then given by

$$
C^{2 c}\left(R^{c}\right)=\sum_{a_{1}, \ldots, a_{2 c}=1}^{p} R^{c}\left(e_{a_{1}}, \ldots, e_{a_{2 c}}\right)\left(e_{a_{1}}, \ldots, e_{a_{2 c}}\right)
$$

where $\left\{e_{1}, \ldots, e_{p}\right\}$ is any orthonormal frame on $P$. We put

$$
k_{2 c}(R)=\frac{1}{c !(2 c) !} \int_{P} C^{2 c}\left(R^{c}\right) d P
$$

where $d P$ is the volume element of $P$. For the case $P \subset E^{n}(K), k_{2 c}\left(R_{P}-R_{K}\right)$ are Weyl's curvature invariants which appear in (1.1).

If $p$ is even, then the Gauss-Bonnet theorem says that

$$
k_{p}\left(R_{P}\right)=(2 \pi)^{p / 2} \chi(P) .
$$

In order to prove (1.1) we need the following lemmas. 
Lemma 2.1. Let $R$ and $S$ be curvature-like tensor fields on a Riemannian manifold $P$. Then

$$
C^{2 c}\left((R+S)^{c}\right)=\sum_{i=0}^{c}\left(\begin{array}{l}
c \\
i
\end{array}\right) C^{2 c}\left(R^{c-i} \wedge S^{i}\right)
$$

Proof. This is a simple consequence of the binomial theorem and Bianchi identities.

The $i$ th power $R_{K}^{i}$ of the curvature operator $R_{K}$ of $E^{n}(K)$ has the following properties.

Lemma 2.2. For any subset $\left\{e_{1}, \ldots, e_{2 i}\right\}$ of an orthonormal frame $\left\{e_{1}, \ldots, e_{n}\right\}$ on $E^{n}(K)$ we have

$$
R_{K}^{i}\left(e_{1}, \ldots, e_{2 i}\right)\left(e_{1}, \ldots, e_{2 i}\right)=K^{i}(2 i) ! / 2^{i}
$$

Furthermore

$$
C^{2 i}\left(R_{K}^{i}\right)=K^{i}((2 i) !)^{2}\left(\begin{array}{c}
p \\
2 i
\end{array}\right) / 2^{i}
$$

Proof. From the definition of the double form multiplication $\wedge([2$, p. 158 (2.2)] $)$ and from the property of $R_{K}$ we have

$$
R_{K}^{i}\left(e_{1}, \ldots, e_{2 i}\right)\left(e_{1}, \ldots, e_{2 i}\right)=K \sum_{\rho \in \operatorname{Sh}(2 i-2,2)} R_{K}^{i-1}\left(e_{\rho_{1}}, \ldots, e_{\rho_{2 i-2}}\right)\left(e_{\rho_{1}}, \ldots, e_{\rho_{2 i}-2}\right)
$$

Here $S h(p, r)$ denote the set of all $(p, r)$ shuffles. By an induction it follows that

$$
R_{K}^{i}\left(e_{1}, \ldots, e_{2 i}\right)\left(e_{1}, \ldots, e_{2 i}\right)=K^{i}\left(\begin{array}{c}
2 i \\
2
\end{array}\right)\left(\begin{array}{c}
2 i-2 \\
2
\end{array}\right) \cdots\left(\begin{array}{l}
4 \\
2
\end{array}\right)=K^{i}(2 i) ! / 2^{i}
$$

Then from (2.1) and (2.5) we obtain (2.6).

Lemma 2.3. For $P \subset E^{n}(K)$ we have

$$
\begin{aligned}
& \left(R_{P}^{c-i} \wedge R_{K}^{i}\right)\left(e_{1}, \ldots, e_{2 c}\right)\left(e_{1}, \ldots, e_{2 c}\right) \\
& \quad=\frac{K^{i}(2 i) !}{2^{i}} \sum_{\rho \in S h(2 c-2 i, 2 i)} R_{P}^{c-i}\left(e_{\rho_{1}}, \ldots, e_{\rho_{2 c-2 i}}\right)\left(e_{\rho_{1}}, \ldots, e_{\rho_{2 c-2}}\right)
\end{aligned}
$$

and 


$$
\begin{aligned}
& C^{2 c}\left(\left(R_{P}-R_{K}\right)^{c-i} \wedge R_{K}^{i}\right) \\
& \quad=\left(\begin{array}{c}
2 c \\
2 i
\end{array}\right)\left(\begin{array}{c}
p-2 c+2 i \\
2 i
\end{array}\right) C^{2 c-2 i}\left(R_{P}^{c-i}\right) C^{2 i}\left(R_{K}^{i}\right) /\left(\begin{array}{c}
p \\
2 i
\end{array}\right)
\end{aligned}
$$

Proof. From the definitions we have

$$
\begin{gathered}
R_{P}^{c-i} \wedge R_{K}^{i}\left(e_{1}, \ldots, e_{2 c}\right)\left(e_{1}, \ldots, e_{2 c}\right) \\
=K \sum_{\rho \in S h(2 c-2,2)}\left(R_{P}^{c-i} \wedge R_{K}^{i-1}\right)\left(e_{\rho_{1}}, \ldots, e_{\rho_{2 c-2}}\right)\left(e_{\rho_{1}}, \ldots, e_{\rho_{2 c-2}}\right) .
\end{gathered}
$$

By an induction it follows that

$$
\begin{aligned}
R_{P}^{c-i} & \wedge R_{K}^{i}\left(e_{1}, \ldots, e_{2 c}\right)\left(e_{1}, \ldots, e_{2 c}\right) \\
& =K^{i}\left(\begin{array}{c}
2 i \\
2
\end{array}\right)\left(\begin{array}{c}
2 i-2 \\
2
\end{array}\right) \cdots\left(\begin{array}{l}
4 \\
2
\end{array}\right) \sum_{\rho \in \operatorname{Sh}(2 c-2 i, 2 i)} R_{P}^{c-i}\left(e_{\rho_{1}}, \ldots, e_{\rho_{2 c}-2 i}\right)\left(e_{\rho_{1}}, \ldots, e_{\rho_{2 c-2 i} i}\right) .
\end{aligned}
$$

This gives (2.7). Next from (2.1) and (2.7) we obtain

$$
\begin{aligned}
& C^{2 c}\left(R_{P}^{c-i} \wedge R_{K}^{i}\right)=\sum_{e_{1}, \ldots, e_{2 c}=1}^{p} R_{P}^{c-i} \wedge R_{K}^{i}\left(e_{1}, \ldots, e_{2 c}\right)\left(e_{1}, \ldots, e_{2 c}\right) \\
& =\sum_{e_{1}, \ldots, e_{2 c}=1}^{p} \sum_{\rho \in \operatorname{Sh}(2 c-2 i, 2 i)} R_{P}^{c-i}\left(e_{\rho_{1}}, \ldots, e_{\rho_{2 c}-2 i}\right)\left(e_{\rho_{1}}, \ldots, e_{\rho_{2 c}-2 i}\right) \\
& \times R_{K}^{i}\left(e_{\rho_{2 c-2 i+1}}, \ldots, e_{\rho_{2 c}}\right)\left(e_{\rho_{2 c-2 i+1}}, \ldots, e_{\rho_{2 c}}\right) \\
& =\sum_{\rho \in S h(2 c-2 i, 2 i)}\left\{\sum_{e_{\rho^{1}}, \ldots, e_{\rho^{2 c}-2 i}=1}^{p} R_{P}^{c-i}\left(e_{\rho_{1}}, \ldots, e_{\rho_{2 c-2 i}}\right)\left(e_{\rho_{1}}, \ldots, e_{\rho_{2 c-2 i}}\right)\right\} \\
& \times\left\{\sum_{e p 2 c-2 i+1, \ldots, e \rho_{2 c}=1}^{p-2 c+2 i} R_{K}^{i}\left(e_{\rho_{2 c-2 i+1}}, \ldots, e_{\rho_{2 c}}\right)\left(e_{\rho_{2 c-2 i+1}}, \ldots, e_{\rho_{2 c}}\right)\right\} \\
& =\left(\begin{array}{c}
2 c \\
2 i
\end{array}\right)\left(\begin{array}{c}
p-2 c+2 i \\
2 i
\end{array}\right) C^{2 c-2 i}\left(R_{P}^{c-i}\right) C^{2 i}\left(R_{K}^{i}\right) /\left(\begin{array}{c}
p \\
2 i
\end{array}\right) \text {. }
\end{aligned}
$$

Now we are ready to prove (1.1). 
Proof of Ishihara's formula (1.1). Let $P \subset E^{n}(K)$ and $\operatorname{dim} P=2 c$ be even. Applying the Gauss-Bonnet theorem (2.3), and Lemmas 2.1, 2.2, 2.3 we have

$$
\begin{aligned}
(2 \pi)^{c} \chi(P) & =k_{2 c}\left(R_{P}\right) \\
& =\frac{1}{c !(2 c) !} \int_{P} C^{2 c}\left(R_{P}^{c}\right) d P \\
& =\frac{1}{c !(2 c) !} \sum_{i=0}^{c}\left(\begin{array}{l}
c \\
i
\end{array}\right) \int_{P} C^{2 c}\left(\left(R_{P}-R_{K}\right)^{c-i} \wedge R_{K}^{i}\right) d P \\
& =\frac{1}{c !(2 c) !} \sum_{i=0}^{c}\left(\begin{array}{l}
c \\
i
\end{array}\right) \int_{P} C^{2 c-2 i}\left(\left(R_{P}-R_{K}\right)^{c-i}\right) C^{2 i}\left(R_{K}^{i}\right) d P \\
& =\sum_{i=0}^{c} K^{i}(2 i-1) ! ! k_{2 c-2 i}\left(R_{P}-R_{K}\right) .
\end{aligned}
$$

\section{Determination of constants $c_{e, i}$}

We refer to [5] for basic facts on non-Euclidean integral geometry and use the notations of $[1,5]$

In this section we determine the constants $c_{e, i}$ of (1.2) by evaluating the integral

$$
A_{e}=\int \mu_{e}\left(S_{o}^{p}(a) \cap g S^{q}(b), K\right) d g,
$$

where $S_{o}^{p}(a)$ is a fixed $p$-dimensional geodesic sphere of radius $a$ in a $(p+1)$-plane $E^{p+1}(K)$ (briefly $E^{p+1}$ ) and $S^{q}(b)$ is a $q$-dimensional geodesic sphere of radius $b$ in $E^{n}(K)$. Note that

$$
\mu_{e}\left(S^{n-1}(a), K\right)=O_{n}\left(\frac{\sin \sqrt{K} a}{\sqrt{K}}\right)^{n-e-1}(\cos \sqrt{K} a)^{e}
$$

We prove the result for the elliptic space $E^{n}(K)$ where $K>0$. The proof for the hyperbolic space $E^{n}(K)$ where $K<0$ is similar. Assume $b<a<(\pi / 6 \sqrt{K})$. We begin with the following lemma.

Lemma 3.1. Let $S_{0}^{m-1}(a)$ be a fixed geodesic sphere of radius $a$ in $E^{m}(K)$ and $S^{m-1}(b)$ the geodesic sphere of radius $b$ with center $x$. Let

$$
B_{e}=\int \mu_{e}\left(S_{o}^{m-1}(a) \cap S^{m-1}(b), K\right) d x
$$


where $d x$ is the volume element of $E^{m}(K)$. Then

$$
\begin{aligned}
B_{e}= & K^{(e / 2)-m+1} \sum_{\substack{0 \leqq i \leqq e \\
i \in \operatorname{even}}} b_{e, m-1-i}(\cos \sqrt{K} a)^{e-i}(\sin \sqrt{K} a)^{m-e-1+i} \\
& \times(\cos \sqrt{K} b)^{i}(\sin \sqrt{K} b)^{m-1+i}
\end{aligned}
$$

where $b_{e, m-1-i}$ are given by

$$
b_{e, m-1-i}=\frac{O_{m-1} O_{m} O_{m+1-i} O_{m+1-e+i}}{O_{m+1} O_{m-e}}\left(\begin{array}{c}
e / 2 \\
i / 2
\end{array}\right)
$$

Proof. Let $\rho$ be the distance of $x$ from the centre of $S_{o}^{m-1}(a)$. Then the radius $y$ of $S_{o}^{m-1}(a) \cap S^{m-1}(b)=S^{m-2}(y)$ is given by

$$
\cos ^{2} \sqrt{K} y=\frac{1}{\sin ^{2} \sqrt{K} \rho}\left(\cos ^{2} \sqrt{K} a+\cos ^{2} \sqrt{K} b-2 \cos \sqrt{K} a \cos \sqrt{K} b \cos \sqrt{K} \rho\right) .
$$

Applying (3.2) and (17.46) in [5, p. 307] we obtain

$$
\begin{aligned}
B_{e}= & K^{(e / 2)-m+1} O_{m} O_{m-1} \int_{-\sin \sqrt{K a} \sin \sqrt{K b}}^{\sin \sqrt{K b}}\left(\cos ^{2} \sqrt{K} a \sin ^{2} \sqrt{K} b+\cos ^{2} \sqrt{K} b \sin ^{2} \sqrt{K} a\right. \\
& -2 u \cos \sqrt{K} a \cos \sqrt{K} b)^{e / 2}\left(\sin ^{2} \sqrt{K} a \sin ^{2} \sqrt{K} b-u^{2}\right)^{(m-e-2) / 2} d u
\end{aligned}
$$

Now from the calculations in $[4, \mathrm{pp} .478-479]$ we get (3.4).

Theorem 3.2. We have

$$
\begin{aligned}
A_{e}= & K^{(e-p-q) / 2} \frac{O_{n+1} \ldots O_{1} O_{p+q-n+3}}{O_{1} O_{p+2} O_{q+2}} \sum_{\substack{0 \leqq i \leqq e \\
i \text { even }}} b_{e, p+q-n+1-i} \frac{O_{q-i+2} O_{p+i-e+2}}{O_{p+q-n+3-i} O_{p+q-n+i-e+3}} \\
& \times(\cos \sqrt{K} a)^{e-i}(\sin \sqrt{K} a)^{p+i-e}(\cos \sqrt{K} b)^{i}(\sin \sqrt{K} b)^{q-i} .
\end{aligned}
$$

Proof. We apply Chern's argument [1, pp. 115-117] to $E^{n}(K)$ and evaluate the integral by iterations. Let $E^{p+1}$ and $E^{q+1}$ be planes which contain $S_{o}^{p}(a)$ and $g S^{q}(b)$ respectively, and let $x$ be the centre of $g S^{q}(b)$. First from the fibering

$$
E_{K}(n) \stackrel{\pi}{\rightarrow}\left\{\left(x, E^{q+1}\right) \mid x \in E^{q+1} \text { unoriented }\right\}
$$


it is not difficult to see that

$$
A_{e}=2 O_{q+1} \ldots O_{2} O_{n-q-1} \ldots O_{2} \int \mu_{e}\left(S_{o}^{p}(a) \cap g S^{q}(b), K\right) d\left(x, E^{q+1}\right),
$$

where

$$
d\left(x, E^{q+1}\right)=d x\left(E^{q+1}\right) \wedge d E^{q+1(n)}
$$

and $d E^{q+1(n)}$ is the density for $(q+1)$-planes in $E^{n}(K)$.

Next we fix $E^{q+1} \subset E^{n}(K)$ and integrate over $d x\left(E^{q+1}\right)$. By transversality we may assume $\operatorname{dim}\left(E^{p+1} \cap E^{q+1}\right)=p+q-n+2$. Let $E^{p+1} \cap E^{q+1}=E^{p+q-n+2}$, and let $E^{n-p-1}=$ $\left(E^{p+q-n+2}\right)^{1}\left(E^{q+1}\right)$ be the complement of $E^{p+q-n+2}$ in $E^{q+1}$ through $x$. A point $x \in E^{q+1}$ can be coordinatized by its projections $x_{1} \in E^{p+q-n+2}$ and $x_{2} \in E^{n-p-1}$. Let $s$ be the distance from $x$ to $E^{p+q-n+2}$. The intersection $g S^{q}(b) \cap E^{p+q-n+2}=S^{p+q-n+1}(\rho) \subset E^{q+1}$ is a geodesic sphere of radius $\rho$, where $\cos \sqrt{K} \rho=\cos \sqrt{K} b / \cos \sqrt{K} s$. Then we have

$$
\begin{aligned}
d x\left(E^{q+1}\right)= & (\cos \sqrt{K} s)^{p+q-n+2} d x_{1}\left(E^{p+q-n+2}\right) \wedge d x_{2}\left(E^{n-p-1}\right) \\
& =K^{(-n+p+2) / 2}(\cos \sqrt{K} s)^{p+q-n+2}(\sin \sqrt{K} s)^{n-p-2} d x_{1} \wedge d s \wedge d u_{n-p-1}
\end{aligned}
$$

where $d u_{n-p-1}$ is the solid angle element such that $\int d u_{n-p-1}=O_{n-p-1}$. Using (3.7) we again calculate the integral by iteration. It follows that

$$
\begin{aligned}
A_{e}= & K^{(-n+p+2) / 2} O_{q+1} \ldots O_{1} O_{n-q-1} \ldots O_{2} O_{n-p-1} \\
& \times \int d E^{q+1} \int_{0}^{b}(\cos \sqrt{K} s)^{p+q-n+2}(\sin \sqrt{K} s)^{n-p-2} d s \\
& \times \int \mu_{e}\left(S_{o}^{p+q-n+1}(r) \cap S^{p+q-n+1}(\rho), K\right) d x_{1}\left(E^{p+q-n+2}\right) .
\end{aligned}
$$

Here $S_{o}^{p+q-n+1}(r)=S_{0}^{p}(a) \cap E^{p+q-n+2}$ is a geodesic sphere of radius $r$ in $E^{p+q-n+2}$, $0 \leqq r \leqq a$. According to (3.4), we obtain, after simple computations,

$$
\begin{aligned}
A_{e}= & K^{(n+e-p-2 q-1) / 2} O_{q+1} \ldots O_{1} O_{n-q-1} \ldots O_{2} \\
& \times \sum_{\substack{0 \leq i \leq e \\
\text { even }}} b_{e, p+q-n+1-i} \frac{O_{q-i+2}}{O_{p+q-n-i+3}}(\cos \sqrt{K} b)^{i}(\sin \sqrt{K} b)^{q-i} \\
& \times \int(\cos \sqrt{K} r)^{e-i}(\sin \sqrt{K} r)^{p+q-n+1+i-e} d E^{q+1} .
\end{aligned}
$$


In order to evaluate the last integral we recall the following lemma due to Chern $[1, \mathrm{p}$. 106].

Lemma 3.3 (Chern). Let $p+q \geqq n$ and $F\left(E^{p}\right)$ be an integrable function which depends only on $E^{p+q-n(q)}=E^{p} \cap E^{q}$, where $E^{q}$ is a fixed q-plane. Then we have

$$
\int F\left(E^{p}\right) d E^{p}=\frac{O_{n+1} \ldots O_{1} O_{p+q-n+1} \ldots O_{1}}{O_{p+1} \ldots O_{1} O_{q+1} \ldots O_{1}} \int F\left(E^{p+q-n(q)}\right) d E^{p+q-n(q)}
$$

Applying (3.9) we obtain

$$
\begin{aligned}
\int(\cos \sqrt{K} r)^{e-i}(\sin \sqrt{K} r)^{p+q-n+1+i-e} d E^{q+1} \\
=\frac{O_{n+1} \ldots O_{p+3}}{O_{q+2} \ldots O_{p+q-n+4}} \int(\cos \sqrt{K} r)^{e-i}(\sin \sqrt{K} r)^{p+q-n+1+i-e} \\
\quad \times d E^{p+q-n+2(p+1)}
\end{aligned}
$$

To integrate over $d E^{p+q-n+2(p+1)}$ let $u$ be the distance of $E^{p+q-n+2}$ from the centre of $S_{o}^{p}(a)$. Then $\cos \sqrt{K} u=(\cos \sqrt{K} a / \cos \sqrt{K} r)$. Since we have

$$
\begin{aligned}
d E^{p+q-n+2(p+1)}= & K^{(-n+q+2) / 2}(\cos \sqrt{K} u)^{p+q-n+2}(\sin \sqrt{K} u)^{n-q-2} \\
& \times d u \wedge d u_{n-q-1} \wedge d E_{[0]}^{n-q-1(p+1)}
\end{aligned}
$$

where $d E_{[0]}^{n-q-1(p+1)}$ is the density for $(n-q-1)$-planes through the origin in $E^{p+1}$, we find, from (17.53b) in [5, p. 309],

$$
\begin{aligned}
& \int(\cos \sqrt{K} r)^{e-i}(\sin \sqrt{K} r)^{p+q-n+1+i-e} d E^{p+q-n+2(p+1)} \\
&= K^{(-n+q+1) / 2} \frac{O_{p+1} \ldots O_{1} O_{p+i-e+2}}{O_{n-q-1} \ldots O_{1} O_{p+q-n+2} \ldots O_{1} O_{p+q-n+i-e+3}} \\
& \times(\cos \sqrt{K} a)^{e-i}(\sin \sqrt{K} a)^{p+i-e} .
\end{aligned}
$$

Combining (3.8), (3.10) and (3.11) we obtain the final result (3.5).

\section{Corollary 3.4 .}

$$
c_{e, e-i}=\frac{O_{n+1} \ldots O_{1} O_{p+q-n+3} O_{q+2-i} O_{p+2-e+i}}{O_{1} O_{p+1} O_{p+2} O_{q+1} O_{q+2} O_{p+q-n+3-i} O_{p+q-n+3-e+i}} b_{e, p+q-n+1-i} .
$$

Remark. (3.5) and (3.12) give (1.4). 


\section{New derivation of Weyl's tube formula for odd-dimensional manifolds in $E^{n}(K)$}

Let $M$ be a compact odd-dimensional imbedded submanifold of $E^{n}(K)$. Denote by $V_{M}^{E^{n}(K)}(r)$ the $n$-dimensional volume of the tube $T(M, r)$ of radius $r$ about $M$ and by $A_{M}^{E^{n}(K)}(r)$ the $(n-1)$-dimensional volume of the boundary of $T(M, r)$. In this section we will derive Weyl's tube formula for $A_{M}^{E^{n}(K)}(r)$ (see (4.4) below) by employing Chern's kinematic formula (1.2) in $E^{n}(K)$ and Ishihara's formula (1.1).

Let $\operatorname{dim} M=2 p+1$. We apply (1.2) with $M$ as a stationary submanifold and with $S^{n-1}(r)$ as the moving submanifold of $E^{n}(K)$. Here $S^{n-1}(r)$ is a geodesic sphere of radius $r$, and $r>0$ is less than or equal to the distance from $M$ to its nearest focal point. Let $x$ be the centre of $g S^{n-1}(r), g \in E_{K}(n)$. Since $E_{K}(n)$ is the semidirect product $E^{n}(K) \dot{\times} S O(n)$ we can write $g S^{n-1}(r)=g_{0} S_{x}^{n-1}(r)$, where $g_{0} \in S O(n)$ and $S_{x}^{n-1}(r)$ denotes the geodesic sphere of radius $r$ with centre $x$. If $d(x, M)>r$, then $M \cap g S^{n-1}(r)$ is empty. Hence we can say that for $0 \leqq e$ even $\leqq 2 p$

$$
\int \mu_{e}\left(M \cap g S^{n-1}(r), K\right) d g=\int_{T(M, r)}\left\{\int_{S O(n)} \mu_{e}\left(M \cap g_{0} S_{x}^{n-1}(r), K\right) d g_{0}\right\} d x,
$$

where $d g_{0}$ is the Haar measure on $S O(n)$ normalized so that $\int_{S O(n)} d g_{0}=O_{n} O_{n-1} \ldots O_{2}$, and $T(M, r)=\left\{x \in E^{n}(K) \mid d(x, M) \leqq r\right\}$. To evaluate the integral (4.1) we may assume $d(x, M)<r$ since the measure of the boundary of $T(M, r)$ is equal to 0 . Then $\chi\left(M \cap g_{0} S_{x}^{n-1}(r)\right)$ is 2 since $M \cap g_{0} S_{x}^{n-1}(r)$ is homeomorphic to an even-dimensional sphere, and

$$
\int_{T(M, r)} d x=V_{M}^{E^{n}(K)}(r)=\int_{0}^{r} A_{M}^{E^{n}(K)}(s) d s
$$

Putting the formulas (1.1) and (1.2) together we obtain from (4.1)

$$
\begin{aligned}
V_{M}^{E^{n}(K)}(r)= & \frac{1}{2 O_{n} \ldots O_{2}(2 \pi)^{p}} \sum_{i=0}^{p}\left\{\sum_{k=0}^{p-i} K^{k}(2 k-1) ! !(2 p-2 k-1) ! !\left(\begin{array}{c}
2 p \\
2 p-2 k
\end{array}\right) c_{2 p-2 k, 2 i}\right. \\
& \left.\times \mu_{2 p-2 k-2 i}\left(S^{n-1}(r), K\right)\right\} \mu_{2 i}(M, K) .
\end{aligned}
$$

According to (3.3), differentiating (4.2) with respect to $r$ and applying

$$
\begin{aligned}
& (2 k-1) ! !(2 p-2 k-1) ! !\left(\begin{array}{c}
2 p \\
2 p-2 k
\end{array}\right)(2 p-2 k-2 i) c_{2 p-2 k, 2 i} \\
& =K(2 k+1) ! !(2 p-2 k-3) ! !(n+1-2 p+2 k+2 i) c_{2 p-2 k-2,2 i}
\end{aligned}
$$

for $0 \leqq k \leqq p-i-1$, we have 


$$
\begin{aligned}
A_{M}^{E^{n}(K)}(r)= & \frac{1}{2 O_{n-1} \ldots O_{2}(2 \pi)^{p}} \sum_{i=0}^{p}(2 p-1) ! !(n-1-2 p+2 i) c_{2 p, 2 i} \mu_{2 i}(M, K) \\
& \times\left(\frac{\sin \sqrt{K} r}{\sqrt{K}}\right)^{n-2-2 p+2 i}(\cos \sqrt{K} r)^{2 p+1-2 i}
\end{aligned}
$$

By a simple calculation we finally get

$$
A_{M}^{E^{n}(K)}(r)=\sum_{i=0}^{p} \frac{O_{n-(2 p+1)+2 i} k_{2 i}\left(R_{P}-R_{K}\right)}{(2 \pi)^{i}}\left(\frac{\sin \sqrt{K} r}{\sqrt{K}}\right)^{n-1-(2 p+1)+2 i}(\cos \sqrt{K} r)^{2 p+1-2 i}
$$

\section{REFERENCES}

1. S. S. Chern, On the kinematic formula in integral geometry, J. Math. Mech. 16 (1966), $101-118$.

2. A. Gray, Some relations between curvature and characteristic classes, Math. Ann. 184 (1970), 257-267.

3. T. IshinaRA, The Euler characteristics and Weyl's curvature invariants of submanifolds in spheres, J. Math. Soc. Japan 39(2) (1987), 247-256.

4. A. Nisenhuis, On Chern's kinematic formula in integral geometry, J. Differential Geom. 9 (1974), 475-482.

5. L. A. SAntalo, Integral Geometry and Geometric Probability (Encyclopedia of Mathematics and its Applications, Vol. 1, Addison-Wesley, 1976).

6. E. Teufel, Anwendung der differentialtopologischen Berechnung der Totalen Krümmung und Totalen Absolutkrümmung in der sphärischen Differentialgeometrie, Manuscripta Math. 32 (1980), 239-262.

7. H. WeYL, On the volume of tubes, Amer. J. Math. 61 (1939), 461-472.

Department of Mathematics and

Mathematics Research Center

Korea Institute of Technology

DAEJEON, 305-701, Korea 